\title{
Study on Turbulence Compensation Surveying and Mapping Algorithm of Ships
}

\author{
Xianmin Wei ${ }^{1}$ and Peng Zhang ${ }^{2}$ \\ ${ }^{1,2}$ School of Computer Engineering, Weifang University \\ 5147 Eastern Dongfeng Street, Weifang 261061, China \\ wfxyweixm@126.com
}

\begin{abstract}
Research on ship photoelectric measuring instrument measurement error compensation technology, which can improve the accuracy of the measurement. According to the ship sailing in the influence of turbulence by the winds and waves, causing airlines direction deflection happen suddenly, and measurement data is not accurate with jumping up and down along the axis. In order to avoid these problems, this paper proposes a kind of ship turbulence measurement compensation algorithm, by adjusting the ship turbulence coefficient to predict the turbulence extend of the ship, and compensate the turbulence measurement error, use the ship turbulence coefficient correction method to revise the result of compensation, overcome turbulence effects impact. The experiment proves that the ship turbulence measurement compensation algorithm compensate measurement results, can improve the accuracy rate of measurement data and satisfactory results have been obtained.
\end{abstract}

Keywords: The ship turbulence, Error compensation, Turbulence coefficient

\section{Introduction}

With the rapid development of ship measurements, a large number of industrial and chemical explorations raised higher accuracy requirements in the field of exploration, therefore, accurate measurement of sea-related data usage, which will help the more efficient use of marine resources, thus promote the measuring vessel industry continuous progress [12]. Among current intelligent mapping methods on ship, the most common way is based on the photoelectric image mapping, ship through the remote collection of marine related image data, after some operations to get accurate results. Ships mapping involves many industries, it is becoming a popular subject of many scholars.

However, some problems exist in their work of traditional mapping methods. When the ship bumps, routes in the horizontal direction, longitudinal axis and perpendicular to the direction of the sea level will occur deflection angle, and the angle of the horizontal direction deflection can cause horizontal coordinate space appear dynamic fluctuations, and longitudinal axis deflection can cause along the longitudinal axis of a Cartesian coordinate system coordinate space appear dynamic fluctuations [3-4]. The dynamic fluctuations dynamic spatial fluctuations and the longitudinal axis and horizontal direction of a Cartesian coordinate system will affect the target image pixel in this coordinate system accuracy. The traditional compensation method is to use coordinate conversion algorithm to compensate measurement error, using a mathematical algorithm to change the Cartesian space coordinate into the reference coordinate system, great turbulence cause the angle deflection in the horizontal direction and the longitudinal axis in great vessels, and ultimately affect the accuracy of the image pixels in the reference coordinate system coordinates, resulting in the 
relatively low error compensation algorithm accuracy rate by using coordinate system. So, how to overcome this turbulence effect on the measurement results has been becoming a problem.

To avoid the above problems, a vessel bump-measuring compensation algorithm was proposed, by adjustment of ship bumping factor, the measurement error resulting from ship turbulence is compensated by using the coefficient correction algorithm of bumping vessels, the compensated result is corrected so as to obtain accurate measurements data [5]. Experiments show that using ship turbulence measuring compensation algorithm for measurement error to compensate, which can effectively improve the accuracy of the measurement data, and achieved satisfactory results.

\section{Ships Mapping Theory}

Ships mapping, which typically uses high-precision surveying instruments, and has dynamic acquisition for ocean data. The principle is as follows:

First, to create a new coordinate system based on the external environment, which expresses optical spatial position of the target image pixel. In the photoelectric measuring device coordinate system, the original spatial location of the target area can be obtained. The new coordinate system and photoelectric measuring device coordinate system coordinates need to be able freely to convert [6-8]. Assuming in the optical measuring device coordinates, the original spatial location of the target can be described, then mathematically, the coordinates can be converted into the new coordinate system. The principles shown in Figure $1:$

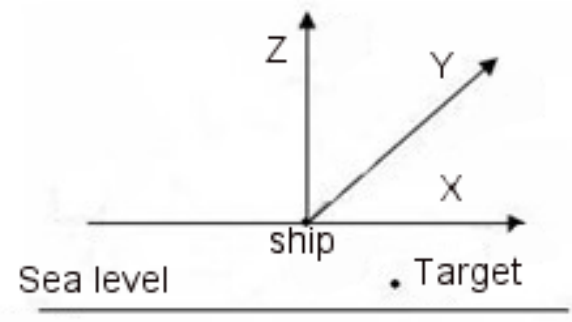

\section{Figure 1. Schematic of Reference Coordinate System}

Figure 1 depicts the new position of a target image pixel coordinates. The starting position of the ship as the coordinate origin, the $X$ axis represents the north and $X-Y$ plane perpendicular to the $\mathrm{Z}$ axis, $\mathrm{Y}$ axis perpendicular to the $\mathrm{X}-\mathrm{Z}$ plane, and then the above coordinate system is defined as the reference coordinate system, by means of $x_{1} t_{1} z_{1}$. Target image pixel position in the reference coordinates was calculated in the formula (1):

$$
\left(x_{1} y_{1} z_{1}\right)=f(\omega, \psi, v, \tau, \sigma, \rho, \vartheta)
$$

Where $\omega$ is the ship's route deflection angle from the horizontal axis, $\psi$ is the longitudinal axis deflection angle of the ship's route, $v$ is the deflection angle of ship's route with the sea-level vertical, $\tau$ is the deflection angle of sea level on horizontal axis when optical measuring device is placed on the ship, $\sigma$ is the deflection angle of sea level on longitudinal axis when optical measuring device is placed on the ship. $\rho$ Is the tilt angle with the sea-level for optical image pixel in the target optical measuring device coordinates, $\vartheta$ is 
the tilt angle with the sea-level for the target area in the target optical measuring device coordinates. Suppose that the function value is known, mathematical methods can be used to obtain objective optical image pixel in the reference coordinate system. Through the abovedescribed formula, to change the coordinates of the target area into the reference coordinate system conversion, the dynamic coefficient is calculated for each axis:

The horizontal axis dynamic fluctuation coefficient is calculated using Eq. (2):

(2)

$$
x^{\prime}=x / \sqrt{\omega^{2}+\psi}
$$

Longitudinal axis dynamic fluctuation coefficient is calculated using Eq. (3):

$$
y^{\prime}=y / \sqrt{\omega^{2}+\psi}
$$

According to the above formula, specific X, Y coordinates can be obtained, complete the basic mapping work.

Through the above known mapping principles, in traditional mapping methods, the final measurement results were severely affected by vessels bumping coefficients. By Eq.(2) and (3) it can be known, when the ship bumps the deflection angle of the horizontal axis along with the longitudinal axis is proportional to dynamic fluctuation coefficient of horizontal and the vertical, and larger the dynamic coefficient of fluctuation, greater errors spatial coordinates values of the Cartesian coordinate system [9]. Therefore, once more substantial jolt occurred, the ship is bound to affect the results of surveying and mapping results, and there is a big error, the accuracy of the mapping is not high.

To avoid the above problems, this paper proposed a turbulence compensation algorithm to continue turbulence factor adjustment, by bumping factor correction algorithm, and to compensate the measurement error of the ship bumping, so as to obtain accurate measurement data [10]. Experimental results show that this method can effectively improve the accuracy of the measurement data, and satisfactory results were achieved.

\section{Ship Bumping Measuring Compensation Algorithm}

\subsection{Ship Bumping Coefficient Adjustment Algorithm}

To compensate the shortcomings of traditional methods, need to compensate measurement error caused by the ship bumping as follows, using Eq.(4) to calculate the coefficient of bumping and get the minimum value of bumping coefficient:

$$
V(k, v)=\beta_{j} R_{j}(k, v)
$$

Bumping coefficient is not fixed, and changes according to the ship's bumping occurrence. If using accelerator device and bumping functions to control the ship, bumping coefficient adjustment can be achieved, and the ship bumping coefficient is adjusted to its minimum.

Although the ship jolt factor regulation theory already exists, but its actual operation process is more complex. Under normal circumstances, a bumpy adjustment function need to be first created, using a recursive algorithm to predict the ship bumping coefficients, so as to predict or forecast ship's bumping conditioning or bumping adjustment coefficient, eventually to compensate measurement error caused by the ship's turbulence. Principle of ship's bumping coefficient adjustment method as shown in Figure 2.

The above described method can compensate the measurement error due to the ship bumping. In Figure 2, $I_{H}(U)$ is the target position parameter, $I_{Q}(U)$ is an optical measuring apparatus antenna parameter, $I_{S}(U)$ is the spatial measurement regulation parameter, 
$J(U)$ is the data transfer function of bumping coefficient regulation, then using equation (5) to calculate the values of transfer function $I_{E N}(U)$ :

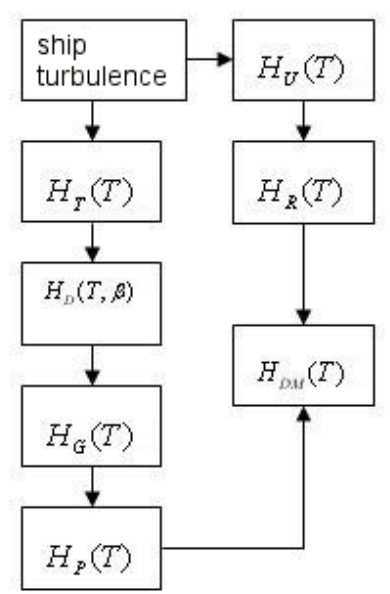

Figure 2. Schematic Ship's Bumping Coefficient Adjustment Method

$H_{D M}(T)=\left(H_{G}(T)+H_{P}(T)+H_{R}(T)\right)^{2} / I(T)$

Where $H_{U}(T)$ is the ship bumping data transfer function, which can be converted into a bumping coefficient governor error $F(u)$ from ship bumping coefficient $W(u)$, this formula is closely related to measuring devices structure mode, optical measuring equipment placement angle and temperature sensitivity, etc.. On the ship. $H_{T}(T)$ Is data transfer function of miniature accelerometer for measuring ship bumping coefficients $W(u)$. $H_{D}(T, \beta)$ is a function of regulating device of ship bumping coefficient to compensate measurement errors caused by the ship, its coefficient $\beta$ can be adjusted according to the actual situation.

Calculating according to the following equation to obtain the results for compensating the bumping measurement errors caused by ship:

$H_{U}(T)=H_{T}(T) H_{D}(T, \beta) / H_{D M}(T)$

Because the accuracy rate of $H_{U}(T)$ is relatively low, so the device can only use regulation to adjust the coefficient $\beta$, so that the variance of measurement error reaches the minimum, and lay foundation to compensate measurement errors caused by ship bumping. To realize the measurement error compensation, turbulence coefficient should first be converted to ship's positional parameters, and the results obtained input into control adjustment circuit.

The main steps of adjusting and predicting as follows:

(1) To optimize the regulation device, thereby suppressing the ship bumping on the accuracy of the measurement data to improve the accuracy of the measurement results obtained.

(2) When the effect of ship bumping on measurement data is smaller than the measurement standard, an adjustment processor is used to compensate the error. 
(3) Calculate the turbulence coefficients and t measurement error, and the calculation result is input to the regulating device, to adjust the adjustment processing results of the processor.

In order to compensate the measurement error due to ship bumping, it needs to predict from the horizontal direction, the vertical axis direction and perpendicular to the direction sea level, and bumping coefficient is closely related to the horizontal and vertical direction error coefficients, so $H_{U}(T)$ is $3 \times 2$ transfer matrix, $H_{D M}(T)$ is $2 \times 2$ transfer matrix, $H_{D}(T, \beta)$ is $3 \times 2$ transfer matrix.

Optimize transfer matrix $H_{D}(T, \beta)$, and through regulation of $H_{D}(T, \beta)$ and $H_{U}(T)$, it is able to make the variance $\tau_{F}^{2}$ of measurement error coefficient caused by ship bumping reach it's the minimum, while can control parameter $\beta$ as reference data.

Optimize the ship bumping adjustment, first need to define $H_{D}(T, \beta)$ as follows:

$$
\left[\begin{array}{ll}
h_{11}\left(t, \beta_{11}\right) & h_{12}\left(t, \beta_{12}\right) \\
h_{21}\left(t, \beta_{21}\right) & h_{22}\left(t, \beta_{22}\right)
\end{array}\right]
$$

Among them:

$$
h_{j k}(t, \beta)=\left(b_{j k} t-c_{j k} t-d_{j k}\right)^{2}, j=0,1 ; k=0,1 ;
$$

Variance $\tau_{F}^{2}$ of measurement error coefficient is the sum of error coefficient from horizontal direction, longitudinal axis and sea level vertical direction, and can be calculated by the formula (8):

$$
\tau_{F}^{2}=\tau_{k F}^{2}
$$

Where $\tau_{k F}^{2}=U^{2} /\left(y_{k}(u)-z_{k}(u)\right)^{2}, u$ is the specified time period, $U$ is specified interval, $y_{k}(u)$ is the error horizontal vector for measurement equipment in the specified direction, $z_{k}(u)$ is the error longitudinal axis vector for the measuring device in the specified direction.

Through the above described it can be learned, coefficient $\beta$ of transfer matrix $\tau_{F}^{2}=\varphi(\beta)$ is calculated as follows:

$$
\beta=\left[b_{j k}, c_{j k}, d_{j k}\right], \quad j=0,1 ; k=1,2 ;
$$

Where $b_{j k}, c_{j k}, d_{j k}$ are known parameter values, $\tau_{F}^{2}$ can be obtained by calculation, in order to gain the minimum of ship bumping coefficient measurement error variance. In the above process, vessels bumping coefficient is the sum from the three direction coefficients, it is possible to obtain the minimum value of these three directions by one calculation. So bumping coefficient is minimum, maximizing inhibition of measurement error due to the turbulence caused by the ship.

Through the ship bumping coefficient adjustment method described above, measurement errors caused by the ship bumping can be effectively compensated. In the above process, however, the ship is in motion, all the parameters calculated above are not fixed, therefore, need real-time compensation to correct results, so as to improve the accuracy of measurement results. 


\subsection{Ship Bumping Factor Correction}

Regulating of ship bumping coefficient to compensate data, first need to get the ship bumps measurement error variance minimum. The ship's jolting factor correction algorithm is able to compensate the results amended.

Output data of ship bumping factor correction algorithm is:

$$
\vartheta(l)=X(l) / \varphi(l)^{2}
$$

Error data of ship bumping coefficient correction algorithm is:

$$
f(l)=\gamma(l)+\vartheta(l)
$$

Weights iterative processing formula of ship bumping factor correction algorithm is:

$$
X(l)=X(l-1)-v_{f}(l) / \varphi(l)
$$

Where: $X(l)=\left(x_{1}(l)+x_{2}(l)+\cdots+x_{m}(l)\right)^{2}$

$$
\varphi(l)=(\gamma(l+1)+\gamma(l+2)+\cdots+\gamma(l+M))^{2}
$$

Expressed mathematically for corrected compensation results described as follows:

$$
\gamma(l)=\log y(l)+\log y(l)^{\prime}
$$

$l=S-2, S-3, \cdots, S-E \quad$ (Input data)

$y(l)=z(l) / e^{k(\vartheta(l))}$ (Compensated output data)

Through the above steps it can be learned, the role of the coefficient correction algorithm of ship bumping is kept to compensate the input data error value of $\vartheta(l)$, thereby correcting the compensation record, and ultimately improve the accuracy of measurement data.

\section{Experimental Results and Analysis}

To determine the effect of the ship bumping coefficient compensation algorithm, this paper designed one comparative experiment. First, the optical measuring device is placed on the level of the ship, manually control course and speed of the ship, so that bumping are made on horizontal direction, longitudinal direction and perpendicular to the horizontal direction. Herein using conventional methods and new methods to get the depth of the target object, the measurement data are recorded in Table 1.

Table 1. Measurement Data using Different Compensation Algorithm

\begin{tabular}{llll}
\hline Method & Horizontal $(\mathrm{m})$ & Longitudinal $(\mathrm{m})$ & Perpendicular $(\mathrm{m})$ \\
\hline The actual data & 1.5 & 3.7 & 5.8 \\
\hline Traditional & 1.1 & 2.9 & 4.9 \\
\hline Proposed & 1.4 & 3.8 & 5.8 \\
\hline
\end{tabular}

According to Table 1, the relevant data can be learned, when the ship bump occurring, using traditional methods and the proposed way to measure, the measurement data obtained are very different. When using the conventional method for the measurement, the measurement data acquired have larger error, and the result of this paper is more accurate. Different measurement accuracy of the compensation algorithm represented in Figure 3. 


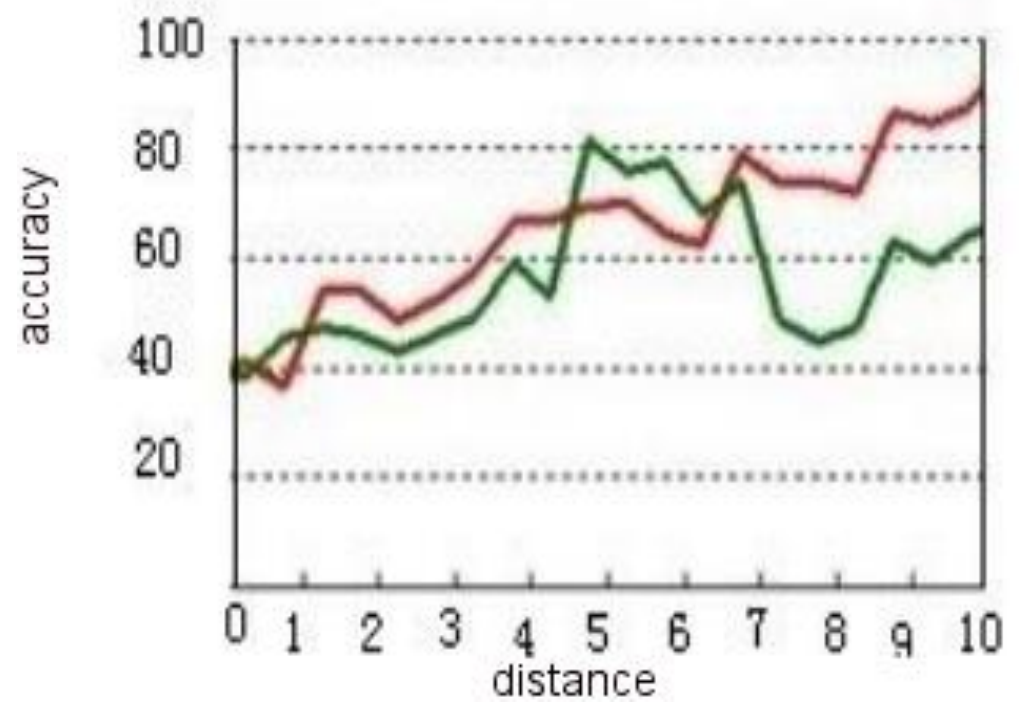

Figure 3. Accuracy of the Results with Different Algorithms

From the above figure, the horizontal axis is measured distance; the vertical axis is the accuracy of the measurement. The upper curve used this proposed method; the curve below used the traditional method. From Figure 3 that, in the event of ship turbulence using the error compensation method of this compensation can improve the accuracy of measurement data. Therefore, using the ship bumping compensation algorithm when the ship turbulence occurs can improve the accuracy of the measurement data, and achieved satisfactory results.

\section{Conclusions}

For the problem during ship suddenly appear thrashing, causing optical measuring equipment acquired data errors, resulting in low data accuracy. Using the conventional method will be affected Cartesian horizontal and vertical dynamic fluctuations, resulting in lower accuracy of the measurement data acquired after the compensation. To avoid these problems, a ship bumping compensation algorithm was proposed to compensate measurement errors, thereby improved the measurement results.

\section{Acknowledgements}

This work is partly supported by Shandong Natural Science Foundation Program (ZR2011FL006), Shandong Science and Technology Development Program (2011YD01044), Shandong Spark Program (2012XH06005), and Weifang municipal Science and Technology Development Program (201301050).

\section{References}

[1] R. Guo, L. Zhong, Z. Wuling and J. Peng, "Research-oriented scheduling jitter motion control", Computer Systems, (2006), p. 7.

[2] J. Wang, S. Gao and N. He, "Shake compensation Implementation of the control system", Xi'an University of Technology, vol. 3, (2009). 
[3] L. Meng, L. G. J. Xinggang, L. D. Xu and F. Fu, "Video jitter correction system motion filtering", Tsinghua University (Natural Science), vol. 1, (2005).

[4] R. Guo, X. Kong, H. Chen and H. Xu, "Research and application of motion control scheduling jitter compensation method", Computer Systems, vol. 2, (2008).

[5] D. Zhao, J. Fang, S. Zhang and Q. Yu, "Phase jitter compensation algorithm in Hopping OFDM systems", Signal processing, vol. 5, (2007).

[6] D. Han, J. Ruan, L. Zhu and F. Guo, "Quantum structure of the photoelectric sensor readout circuit imaging system", Computer Measurement \& Control, vol. 3, (2011).

[7] X. Ning, A. Zhang and J. He, "Study of airborne photoelectric detection simulation system", Computer simulation, vol. 10, (2007).

[8] C. Canudas de Wit, "Robust, Control for Servo-Mechanisms Under Inexact Friction Compensation", Automatica, vol. 29, no. 3, (1993).

[9] J. Amin, B. Friedland and A. Harnoy, "Implementation of a Friction Estimation and Compensation Technique”, IEEE Control Systems, vol. 8, (1997).

[10] K. Li and J. Baillieul, "Robust Quantization for digital finite Communication Bandwidth Control", IEEE Transaction on Automatic Control, vol. 49, no. 9, (2004).

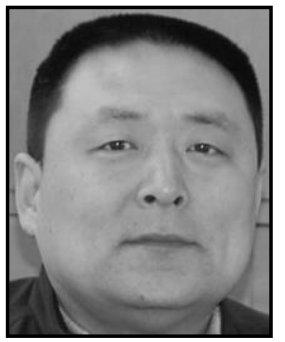

Xianmin Wei, He received the $M$. Sc. degree in computer applications from Shandong Science and Technology University (2005). $\mathrm{He}$ is currently an associate professor in school of computer engineering at Weifang University, China. He has published over 30 papers and 3 books in professional fields. Since 2011, he has been a member of IEEE$\mathrm{CS}, \mathrm{ACM}$ and CCCF, respectively. His fields of research are focused on swarm intelligent, intelligent sensor networks.

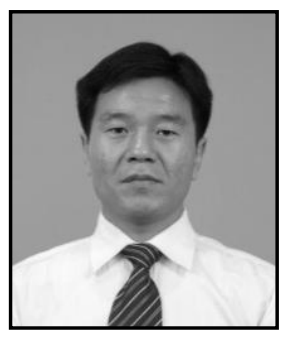

Peng Zhang, He received his $\mathrm{PhD}$ degree of communication and information systems from Shandong University, Jinan, P. R. China, in December 2012. Currently, he is a lecturer in the School of Computer Engineering, Weifang University, Shandong, and P. R. China. His current research interests focus on advanced coded modulation, massive MIMO, spatial modulation, cross-layer design, cooperative communications and 4G/B4G wireless communications. 